\title{
Novae In External Galaxies From The POINT-AGAPE Survey And The Liverpool Telescope
}

\author{
M. J. Darnley*, M. F. Bode*, E. J. Kerins* and T. J. O’Brien ${ }^{\dagger}$ \\ ${ }^{*}$ Astrophysics Research Institute, Liverpool John Moores University, Birkenhead, CH41 1LD, UK \\ ${ }^{\dagger}$ Jodrell Bank Observatory, The University of Manchester, Macclesfield, SK11 9DL, UK
}

\begin{abstract}
We have recently begun a search for Classical Novae in M31 using three years of multicolour data taken by the POINT-AGAPE microlensing collaboration with the $2.5 \mathrm{~m}$ Isaac Newton Telescope (INT) on La Palma. This is a pilot program leading to the use of the Liverpool Telescope (LT) to systematically search for and follow novae of all speed classes in external galaxies to distances up to around $5 \mathrm{Mpc}$.
\end{abstract}

\section{INTRODUCTION}

The importance of the study of novae in external galaxies has been recognised since the time of Hubble [1]. However, there are great difficulties in obtaining the frequency and duration of observations with large enough telescopes to determine the peak magnitude and speed class for a meaningful sample of objects (see papers by Shafter, Shara and Della Valle, this volume).

We aim to search for and follow the temporal development of novae across a range of speed classes in selected galaxies over several years. Our goals are to tighten the MMRD, $t_{15}$ and other relationships, which make classical novae potentially very important distance indicators [2]. We hope to help resolve the debate about the dependence of nova rate with galaxy type and stellar population [3, \#]. Finally, we wish to determine whether there are indeed two distinct populations of classical novae [5] and how this might relate to current models of the outburst.

This project is being pushed on two fronts: first with existing data from the POINTAGAPE gravitational microlensing survey and then with the soon-to-be-commissioned Liverpool Telescope (LT). We plan to automate as far as possible the data reduction and novae selection processes.

\section{Identification of Novae}

We are identifying novae using a method similar to that outlined in Shafter et al [6]. The images are first carefully aligned and then have their point-spread functions matched. We identify strongly varying sources by subtracting the aligned and matched 


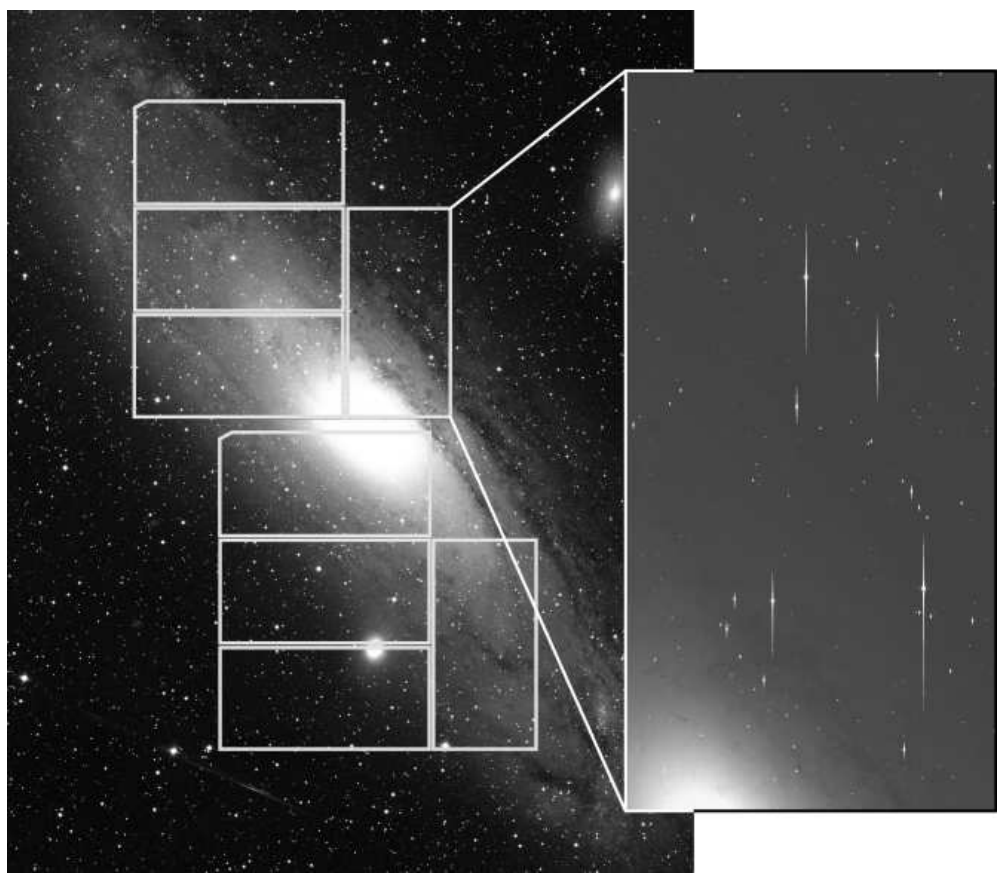

FIGURE 1. The Andromeda Galaxy and the positions of the two POINT-AGAPE fields. (Image adapted from an original image obtained by Bill Schoening, Vanessa Harvey/REU programme/AURA/NOAO/NSF.) Inset shows one of the four chips from one of our INT WFC frames.

images from one another and searching for any residual sources. Photometry is carried out on the data using the DAOPHOT package within the IRAF environment.

In this dataset we are sensitive to objects down to $r^{\prime} \simeq 23$, though our sensitivity is a strong function of position and decreases towards the bulge. Ultimately we plan to use simulated datasets to correct for this spatial bias.

\section{THE POINT-AGAPE SURVEY}

POINT-AGAPE is an Anglo-French collaboration, which is searching for gravitational microlensing events against the mostly unresolved stars in M31 [7]. The survey team is employing a modified detection technique dubbed "pixel lensing" developed to cope with the problems associated with temporal variations in seeing and sky background. The project has evolved from the pilot AGAPE (Andromeda Galaxy Amplified Pixels Experiment) programme conducted at Pic du Midi, which probed the M31 bulge [8]. POINT-AGAPE is using the wide-field camera (WFC) on the INT to survey a much larger area (POINT is an acronym for Pixel-lensing Observations with the INT). Since autumn 1999, two fields covering 0.6 square degrees of the M31 disk have been monitored regularly in three colours (Sloan-like $g^{\prime}, r^{\prime}$ and $i^{\prime}$ filters, which correspond to wavelengths of 486, 622 and $767 \mathrm{~nm}$, respectively). The locations of the two fields are shown in Figure 11. To date three seasons of data comprising roughly 180 epochs have been collected. 

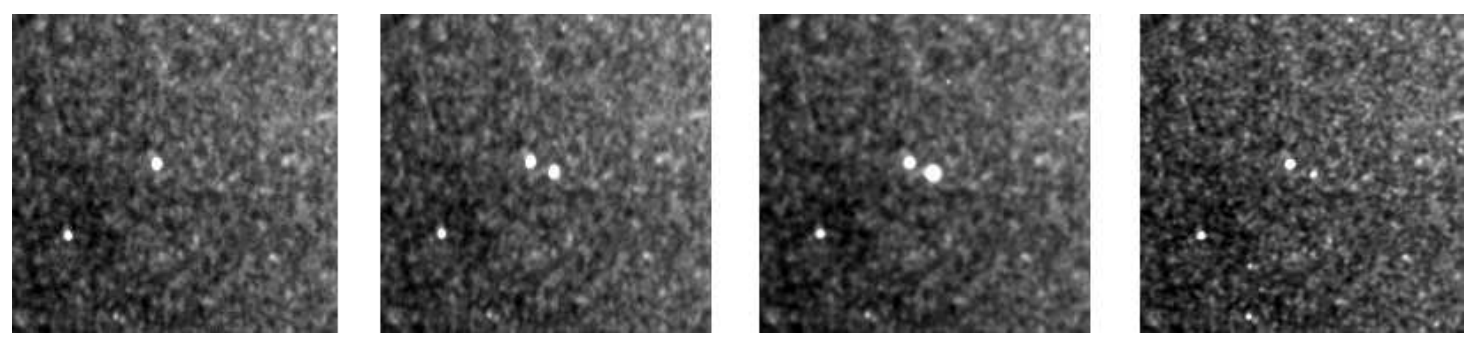

FIGURE 2. An $r^{\prime}$-band image sequence of the first nova candidate from the POINT-AGAPE INT survey.

The prerequisites for the detection of microlensing are good temporal sampling and a long baseline of observations and it is these same qualities that make the POINT-AGAPE dataset an excellent repository for novae searches. Since novae are much brighter than typical microlensing events they should, for the most part, show up as resolved sources, negating the need for us to employ the pixel-lensing method.

However, with around 50GB of data to analyse, the task of finding and cataloging novae in M31 remains a challenging one. To meet this challenge we are developing algorithms to automatically reduce the data and search for novae. Parts of this software have already allowed us to discover two probable novae candidates in a small subset of the dataset provided by the POINT-AGAPE collaboration.

\section{First Results}

We have identified two nova candidates in the POINT-AGAPE dataset. Both novae were initially discovered in the $r^{\prime}$-band data and then located in both the $g^{\prime}$ and $i^{\prime}$ bands. The first nova candidate became visible on 7th September 1999, was caught during the initial rise in both $r^{\prime}$ and $g^{\prime}$ bands, and was subsequently followed to provide an estimate of $t_{2} \simeq 20$ days, classing this candidate as a fast nova [9]. Figure 2 shows a sequence of $r^{\prime}$-band images which straddle the outburst. The second nova was discovered near maximum at the beginning of the first observing season on 2nd August 1999. We estimate $t_{2} \simeq 30$ days for this event, making it a moderately fast nova [9]. Figures 3 and 7 show the $r^{\prime}$-band and $i^{\prime}$-band lightcurves for the first and second nova candidates, respectively. The photometric calibration of these lightcurves is still being worked on, but these results illustrate the types of nova observations that we will be able to secure.

The two novae candidates have been found whilst testing our detection methods on a small 1024x1024 pixel section of the data over a period of about three months, amounting to less than $0.3 \%$ of the entire dataset. We are now beginning to search for variable objects in the whole dataset and, based on estimates of the M31 novae rate of $29 \pm 4 \mathrm{yr}^{-1}$ [10], we are expecting to find 30-50 classical novae. One of the advantages of our dataset is excellent temporal coverage which should allow us to produce reasonably complete lightcurves, hopefully with many sampling the all-important time of maximum light. 


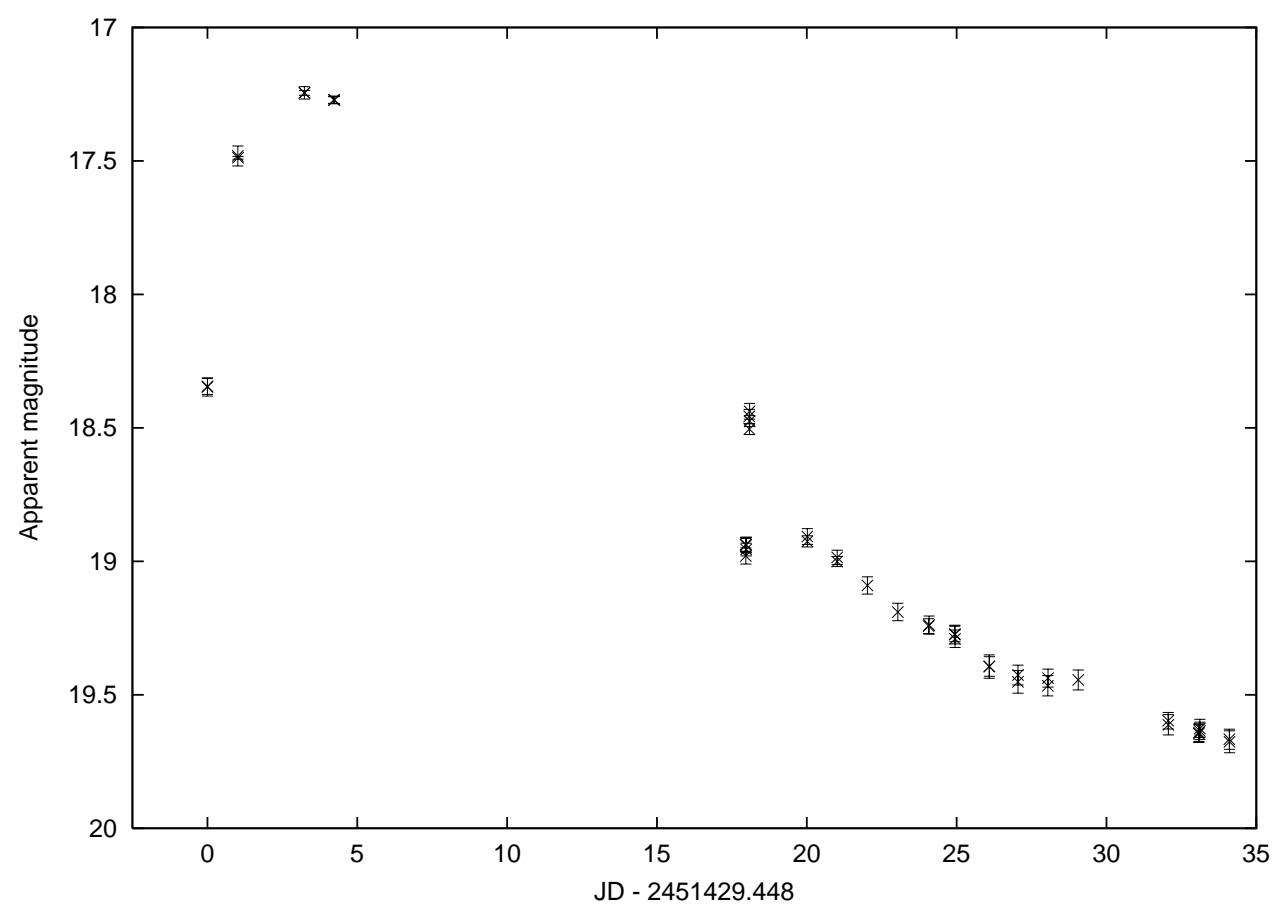

FIGURE 3. $\quad r^{\prime}$-band lightcurve of the first nova candidate from the POINT-AGAPE survey data.

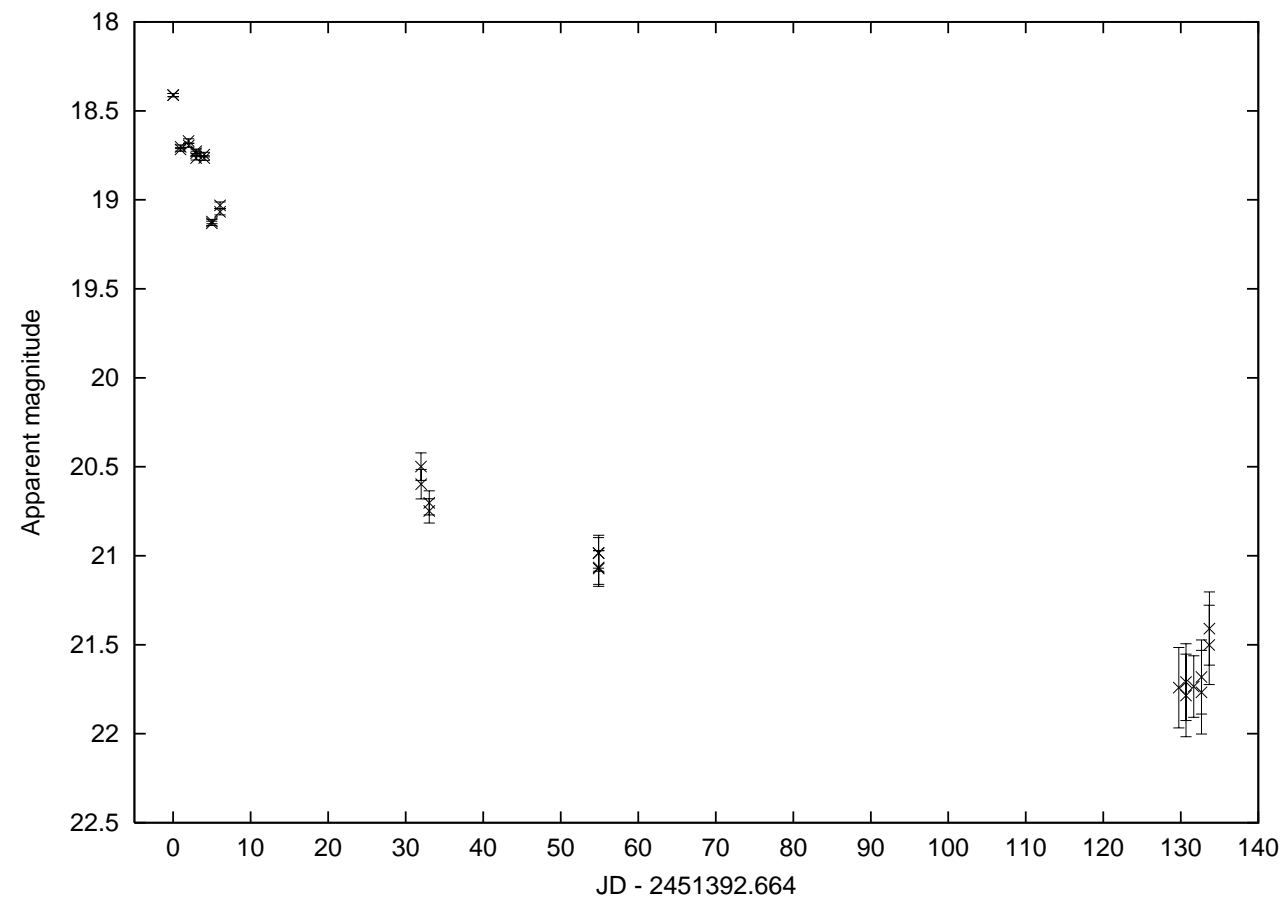

FIGURE 4. $i^{\prime}$-band lightcurve of the second nova candidate from the POINT-AGAPE survey data. 


\section{THE LIVERPOOL TELESCOPE}

The Liverpool Telescope, with a primary mirror diameter of $2 \mathrm{~m}$, will be the World's largest fully-robotic telescope. The LT is currently being reassembled at the Observatorio del Roque de Los Muchachos, La Palma. Its primary instruments are a CCD camera with a 12-position filter wheel, a near-IR camera and a fibre-fed spectrograph. The telescope is able to move rapidly to acquire targets and will have arc-second pointing.

The main scientific goals of the LT are: (i) the monitoring of variable objects on all timescales, from seconds to years; (ii) the ability to rapidly react to unpredictable phenomena and their systematic follow up; (iii) simultaneous or coordinated observations with other facilities, both on the ground and in space; and (iv) small-scale surveys and serendipitous source follow-up. Further details about the LT can be found at the telescope's website (http://telescope.livjm.ac.uk).

Guaranteed time has been approved for our novae in external galaxies program and the telescope is expected to see first light in the autumn of 2002. We will use the unique capabilities of the LT to systematically monitor a carefully selected set of external galaxies; M81, M64 and M94, to discover and follow as complete a sample of novae as possible in each system. We shall be able to detect objects down to $m_{V} \approx 24$. Over a three-year programme, we will discover more classical novae than have been discovered in our own Galaxy to date, and in many cases provide better lightcurve and distance determinations. A by-product of the survey will be the discovery of many other luminous variables in our target galaxies.

\section{ACKNOWLEDGMENTS}

We wish to thank the POINT-AGAPE collaboration for use of its data. MJD would like to thank Andy Newsam for help with preparing some of the figures. MJD's research is supported by a PPARC PhD studentship.

\section{REFERENCES}

1. Hubble E., 1929, ApJ, 69, 103

2. Warner B., 1989, Classical Novae, J. Wiley \& Sons, p8

3. Della Valle, M., Rosino, L., Bianchini, A., Livio, M., 1994, A\&A, 287, 403

4. Yungelson, I., Livio, M., Tutukov, A., 1997, ApJ, 481, 127

5. Della Valle, M., Bianchini, A., Livio, M., Orio, M., 1992, A\&A, 266, 232

6. Shafter A.W., Ciardullo R., Pritchet C. J., 2000, ApJ, 530, 193

7. Kerins E.J. et al., 2001, MNRAS, 323, 13

8. Ansari R. et al., 1997, A\&A, 324, 843

9. Payne-Gaposchkin, C., 1957, The Galactic Novae, North-Holland

10. Capaccioli M., Della Valle M., D’Onofrio M., Rosino L., 1989, AJ, 97, 1622 